PROCEEDINGS OF THE

AMERICAN MATHEMATICAL SOCIETY

Volume 89, Number 1, September 1983

\title{
RATIONAL CURVE WITH ONE CUSP
}

\author{
HISAO YOSHIHARA
}

\begin{abstract}
In this paper we shall give new examples of plane curves $C$ satisfying $C-\{P\} \cong \mathbf{A}^{\prime}$ for some point $P \in C$ by using automorphisms of open surfaces.
\end{abstract}

1. In this paper we assume the ground field is an algebraically closed field of characteristic zero. Let $C$ be an irreducible algebraic curve in $\mathbf{P}^{2}$. Then let us call $C$ a curve of type I if $C-\{P\} \cong \mathbf{A}^{1}$ for some point $P \in C$, and a curve of type II if $C \backslash L \cong \mathbf{A}^{1}$ for some line $L$. Of course a curve of type II is of type I. Curves of type II have been studied by Abhyankar and Moh [1] and others. On the other hand, if the logarithmic Kodaira dimension of $\mathbf{P}^{2}-C$ is $-\infty$ or the automorphism group of $\mathbf{P}^{2}-C$ is infinite dimensional, then $C$ is of type $\mathrm{I}[3,6]$. So the study of type I seems to be important, but only a few examples are known that are of type I and not of type II. Moreover the definitions of those examples are not so plain [4,5]. Here we shall give new examples by using automorphisms of open surfaces.

2. First we recall the properties of type I. Let $\left(e_{1}, \ldots, e_{t}\right)$ be the sequence of the multiplicities of the infinitely near singular points of $P$ in case $C$ is of type $\mathrm{I}$ with degree $d \geqslant 3$. Then put

$$
R=d^{2}-\sum_{i=1}^{t} e_{i}^{2}-e_{t}+1 .
$$

Since a curve of type II is an image of a line by some automorphism of $\mathbf{A}^{2}$ [1], we see that $R \geqslant 2$ for this curve by the same argument as below. Denoting by $G$ the automorphism group of $\mathbf{P}^{2}-C$, we have $G \supset \mathbf{A}^{m}$ for all $m \geqslant 1$ if $R>0$, and $G$ is a finite group if $R<0$ [6]. One of the conditions for type I curves to be type II is the following:

Lemma 1. Suppose C is type I. Then it is type II if and only if $d=e_{1}+e_{2}$. (If $t=1$, we put $e_{2}=1$.)

Proof. If $C$ is type II, then it is a birational transform of a line [1]. Hence we have $d<e_{1}+e_{2}+e_{3}$, which is the inequality of M. Noether [2]. Moreover since $C$ is type II, we have $d=I_{P}(C, L)$, which is the intersection number of $C$ and $L$ at $P$. Hence we conclude $d=e_{1}+e_{2}$.

Conversely, assume $d=e_{1}+e_{2}$. Note that we have, in general, $d \geqslant I_{P}(C, L) \geqslant e_{1}$ $+e_{2}$, where $L$ is the tangent at $P$. Thus we have $d=I_{P}(C, L)$, which implies $C \cap L=\{P\}$, i.e., $C$ is type II.

Received by the editors November 16, 1982.

1980 Mathematics Subject Classification. Primary 14H45.

(C) 1983 American Mathematical Society 0002-9939/83/0000-1455/\$01.75 
Now we give examples. Let $(X, Y, Z)$ be homogeneous coordinates on $\mathbf{P}^{2}$, and let $L$ be the line defined by $Z=0$. Let $f$ be a nonlinear automorphism of $\mathbf{P}^{2}-L$ and $P$ the point of the indeterminacy of the birational transformation $\bar{f}$ defined by $f$. Let $H$ be a line not passing through $P$, and put $\Delta=\bar{f}(H)$ and $n=\operatorname{deg} \Delta$. Then we have $n \geqslant 2$. Of course $\Delta \backslash L \cong \mathbf{A}^{1}$ and $\operatorname{Aut}\left(\mathbf{P}^{2}-\Delta\right)$ is an infinite-dimensional group.

LEMMA 2. Any automorphism $\varphi$ of $\mathbf{P}^{2}-\Delta$ induces an automorphism of $L$ fixing the point $P$ if $n \geqslant 3$.

Proof. We consider the elimination of the point of the indeterminacy of the birational transformation $\bar{\varphi}$ defined by $\varphi$. Let $\sigma_{i}, i=1, \ldots, r$, be the blow-ups such that the total transform $\sigma^{-1}(\Delta)$, where $\sigma=\sigma_{1} \cdots \sigma_{r}$, has normal crossings. Then the centers of them are unique if the number $r$ is minimal. By Lemma 1 we see that $\left(\sigma_{1} \sigma_{2}\right)^{-1}[L]$ does not meet $\left(\sigma_{1} \sigma_{2}\right)^{-1}[\Delta]$, where [ ] denotes the proper transform. Since $r \geqslant 3$, we infer that $\bar{\varphi}[L]$ is also a line, hence it coincides with $L$.

Let $\Gamma$ be a line or an irreducible conic meeting $\Delta$ at only one point different from $P$. Let $g$ be a nonlinear automorphism of $\mathbf{P}^{2}-\Delta$, and let $\bar{g}$ be the birational transformation defined by $g$. In case $n=2$ we assume $\bar{g}$ induces an automorphism of $L$. Then put $C=\bar{g}(\Gamma)$. By the lemmas we see that $C-\{P\} \cong \mathbf{A}^{1}$ and $C$ is not type II. Let $\sigma$ be the composition of the blow-ups such that the number of them is the smallest one to obtain a morphism $\bar{g} \sigma$. Since the morphism $\bar{g} \sigma$ first contracts the proper transform $\sigma^{-1}[\Delta]$, we have $e_{t}=n$ [resp. 2n] and $d^{2}-\sum_{i=1}^{t} e_{i}^{2}=1$ [resp. 4] if $\operatorname{deg} \Gamma=1$ [resp. 2]. Hence $R=2-n$ [resp. $5-2 n$ ] if $\operatorname{deg} \Gamma=1$ [resp. 2]. Moreover we have $I_{P}(C, L)=d-1$ [resp. $d-2$ ] if $\operatorname{deg} \Gamma=1$ [resp. 2].

3. Here we give the examples more concretely. In case $\Gamma$ is a line, let $\Delta$ be the curve defined by $Y Z^{n-1}=X^{n}$. Then $\Gamma$ is defined by $Y=0$. Now, Wakabayashi shows the following: let $\bar{g}(X, Y, Z)=\left(g_{1}, g_{2}, g_{3}\right)$. Then

$$
\begin{aligned}
& g_{1}=a X\left(Y Z^{n-1}-X^{n}\right)^{m n}+\sum_{i=1}^{m} b_{i} Z^{n+1}\left(Y Z^{n-1}-X^{n}\right)^{m n-i}, \\
& g_{2}=Z^{-(n-1)}\left[\begin{array}{l}
a^{n}\left(Y Z^{n-1}-X^{n}\right)^{m n+1} \\
\quad+\left\{a X\left(Y Z^{n-1}-X^{n}\right)^{m}+\sum_{i=1}^{m} b_{i} Z^{n i+1}\left(Y Z^{n-1}-X^{n}\right)^{m-i}\right\}
\end{array}\right.
\end{aligned}
$$

and

$$
g_{3}=Z\left(Y Z^{n-1}-X^{n}\right)^{m n}
$$

where $a \neq 0, m \geqslant 1$ and $b_{i}$ are arbitrary for $i=1, \ldots, m$. Thus the curve defined by $g_{2}=0$, where $m \geqslant 1$ and $n \geqslant 2$, is the required one.

On the contrary, in case $\Gamma$ is a conic, let $\Delta$ and $\Gamma$ be the curves defined by $Y Z-X^{2}=0$ and $Y Z-X^{2}+a Y^{2}=0$, where $a \neq 0$, respectively.

By these examples we know that every integer $\leqslant 1$ is taken as the value $R$. Finally we raise the following:

Problem. If $C$ is type I with $R \geqslant 2$, then is it true that $C$ is type II? 


\section{REFERENCES}

1. S. S. Abhyankar and T. T. Moh, Embeddings of the line in the plane, J. Reine Angew. Math. 276 (1975), 148-166.

2. J. L. Coolidge, $A$ treatise on algebraic plane curves, Dover, New York, 1959.

3. S. Iitaka, Algebraic geometry, an introduction to birational geometry of algebraic varieties, Graduate Texts in Math., 76, Springer-Verlag, Berlin and New York, 1981.

4. S. Tsunoda, The structure of open algebraic surfaces and its application to plane curves, Proc. Japan Acad. Ser. A. Math. Sci. 57 (1981), 230-232.

5. H. Yoshihara, On plane rational curves, Proc. Japan Acad. Ser. A Math. Sci. 55 (1979), 152- 155.

6. Projective plane curves and the automorphism groups of their complements (to appear).

Department of Mathematics, Faculty of General Education, Niigata University, 950-21 NiIgATA, JAPAN 